\title{
EDITORIAL
}

\section{Genome editing as a promising tool to address global food security}

World population is expected to rise to 9 billion by 2050 . According to the Food and Agriculture Organization of the United Nations, agricultural production needs to increase by 60 to $70 \%$ by then. Transgenic technology became a promising solution to address the global food security in the 1990s. However, currently, transgenic crops are not encouraged in many countries mainly due to health and environmental concerns. Recent advancements in DNA sequencing and genome editing technologies have opened up new possibilities to address the drawbacks of transgenic approaches and overcome the limitations of conventional plant breeding. On the other hand, genome editing technologies can be used to improve crops without introducing foreign genes, and these technologies will not face the high regulatory hurdles faced by transgenic technology. Genome editing technologies such as zinc finger nuclease (ZFNs) and transcription activator-like effector nucleases (TALENs) allowed plant scientists to precisely target and edit any gene of interest. CRISPRCas9, short for clustered regularly interspaced short palindromic repeats and CRISPR-associated protein 9, is the most recently introduced genome editing technique which is much cheaper and efficient compared to the previous genome editing techniques. Bacterial CRISPRCas system was first identified in prokaryotes and further developed as a tool to be used in mammalian and plant genome editing.

CRISPR-Cas9 system is basically an engineered Cas9 nuclease fused with a guide RNA (gRNA) (Lino et al., 2018). The guide RNA and Cas9 protein complex target the sequence of interest and cause a double strand DNA break. Scientists can then mimic this DNA repair mechanism of the cell to join the cut ends by adding or deleting one or few base pairs causing mutations or an existing DNA segment can be replaced with a new DNA segment. Sequencing technologies have rapidly improved over the last few decades, and the sequencing cost per $\mathrm{Mb}$ has dropped from over \$5 000 in 2001 to \$0.06 in 2013. Sequencing information is required to design CRISPRCas9 vectors targeting specific genomic sites. CRISPR has been used for genome editing in various crop species such as rice, wheat, maize, cotton, grapes and carrot over the last two decades (Li et al., 2016; Shi et al., 2017; Howells et al., 2018). Most of these studies were mainly aimed at increasing crop yield, disease resistance, and tolerance to biotic and abiotic stresses.

Crop yield is a quantitative trait controlled by many genes; however, some genes negatively affect yield. CRISPR-Cas9 system can be used to specifically knock out genes that negatively affect yield to improve crop production. In rice, CRISPR-Cas9 system has been used to specifically knock out $L A Z Y 1$ gene to increase tiller number and yield (Miao et al., 2013). Multiple gene knockouts have also been achieved by using a CRISPR-Cas9-mediated multiplex genome-editing system and knocked out three genes that negatively affect rice grain weight ( $\mathrm{Xu}$ et al., 2016). Further, CRISPR-Cas9-mediated genome-wide screening is a promising tool to target multiple genes using a pool of gRNAs.

CRISPR-Cas9 system has been successfully used to increase plant resistance to viral, fungal and bacterial pathogens. For example, tomato germplasm resistance to powdery mildew has been developed targeting MILDEW RESISTANT LOCUS O (Nekrasov et al., 2017). Another example demonstrates the engineering of CRISPR-Cas9mediated potyvirus resistance in Arabidopsis. In most cases, CRISPR-Cas9 system has been used to target host factors. However, some studies have shown that this system can be used to target viral genomes directly (Sedeek et al., 2019) suggesting many opportunities to manage viral disease either plant or animal origin.

Abiotic stresses such as drought, cold and salinity adversely affect plant growth and development and reduce yield. Recently, a drought-tolerant maize variety has been developed by editing a gene encodes maize negative regulator of ethylene responses, using CRISPRCas9 system (Shi et al., 2017). Numerous potential genes involved in abiotic stress tolerance have been identified with the recent advancements in DNA sequencing technologies, and CRISPR-Cas9 system is a valuable tool to edit these genes and develop crops that are climate insensitive.

CRISPR-Cas9 system has been used successfully for targeted improvement of many other crop traits such as enhancing plant herbicide resistance (Butler et al., 2015; Li et al., 2016), enhancing yield quality (Sun et al., 2017; Yu et al., 2017), and morphological traits (Li et al., 2018). The application of DNA sequencing and genome editing technologies to improve crops and develop high-yielding, high-quality crops. In Sri Lanka, the first dedicated Biotechnology laboratory was established at the Plant Genetic Resources Centre at the Department of Agriculture in 1987 with the help of the Japan International Cooperation Agency (JICA). However, the progression and adaptation of biotechnology research has been extremely slow in the country due to severely limited funding for research and low availability of trained human resources. For these reasons, Sri Lanka has not yet capitalised on the existing variations in biodiversity of many food crops including 
rice to improve yield and quality. As we move towards a biotechnological era, cheap, readily available technologies like next generation sequencing (NGC) and CRISPR-Cas9 need to be adopted to improve crop yield gap and thus the country's food security.

Saman Seneweera

Email:Saman.se@nifs.ac.lk

\section{References}

Butler, N.M., Atkins, P.A., Voytas, D.F. and Douches, D.S. (2015). Generation and inheritance of targeted mutations in potato (Solanum tuberosum L.) using the CRISPR/Cas system. PloS one 10(12), e0144591.

Howells, R.M., Craze, M., Bowden, S. and Wallington, E.J. (2018). Efficient generation of stable, heritable gene edits in wheat using CRISPR/Cas9. BMC plant biology 18(1): 215.

Li, J., Meng, X., Zong, Y., Chen, K., Zhang, H., Liu, J., et al. (2016). Gene replacements and insertions in rice by intron targeting using CRISPR-Cas9. Nature plants 2(10): 16139.

Li, T., Yang, X., Yu, Y., Si, X., Zhai, X., Zhang, H., et al. (2018). Domestication of wild tomato is accelerated by genome editing. Nature biotechnology.

Lino, C.A., Harper, J.C., Carney, J.P. and Timlin, J.A. (2018). Delivering CRISPR: a review of the challenges and approaches. Drug delivery 25(1): 1234-1257.

Miao, J., Guo, D., Zhang, J., Huang, Q., Qin, G., Zhang, $\mathrm{X}$, et al. (2013). Targeted mutagenesis in rice using CRISPR-Cas system. Cell research 23(10): 1233.

Nekrasov, V., Wang, C., Win, J., Lanz, C., Weigel, D. and Kamoun, S. (2017). Rapid generation of a transgenefree powdery mildew resistant tomato by genome deletion. Scientific reports 7(1), 482.

Sedeek, K.E., Mahas, A., and Mahfouz, M. (2019). Plant genome engineering for targeted improvement of crop traits. Frontiers in plant science 10.

Shi, J., Gao, H., Wang, H., Lafitte, H.R., Archibald, R.L., Yang, M., et al. (2017). ARGOS 8 variants generated by CRISP-Cas9 improve maize grain yield under field drought stress conditions. Plant biotechnology journal 15(2): 207-216.

Sun, Y., Jiao, G., Liu, Z., Zhang, X., Li, J., Guo, X., et al. (2017). Generation of high-amylose rice through CRISPR/Cas9-mediated targeted mutagenesis of starch branching enzymes. Frontiers in plant science 8: 298.

Xu, R., Yang, Y., Qin, R., Li, H., Qiu, C., Li, L., et al. (2016). Rapid improvement of grain weight via highly efficient CRISPR/Cas9-mediated multiplex genome editing in rice. Journal of genetics and genomics $=Y i$ chuan xue bao 43(8): 529.

Yu, Q.-h., Wang, B., Li, N., Tang, Y., Yang, S., Yang, T., et al. (2017). CRISPR/Cas9-induced targeted mutagenesis and gene replacement to generate long-shelf life tomato lines. Scientific reports 7(1): 11874. 